\title{
RETRACTION
}

\section{Growth of single-walled carbon nanotube at a low temperature by alcohol catalytic chemical vapor deposition using Ru catalysts - RETRACTION}

Takayuki Fujii, Takuya Okada, Takahiro Saida, Shigeya Naritsuka, and Takahiro Maruyama

doi: 10.1557/adv.2018.26, Published by Materials Research Society, 10 January 2018.

As a result of a technical error at the Publisher, the same article was erroneously published twice online. It is therefore necessary to retract the second appearance of the article in favor of the first to maintain a single version of record, though there is no issue with the article itself. The retracted article will only publish in the online edition of its assigned volume. Cambridge University Press sincerely apologizes to the authors and readers for this mistake.

\section{REFERENCES}

1. T. Fujii, T. Okada, T. Saida, S. Naritsuka, and T. Maruyama (2018). Growth of single-walled carbon nanotube at a low temperature by alcohol catalytic chemical vapor deposition using Ru catalysts. $M R S$ Advances, 1-7. doi:10.1557/adv.2018.4.

2. T. Fujii, T. Okada, T. Saida, S. Naritsuka, and T. Maruyama (2018). RETRACTED - Growth of single-walled carbon nanotube at a low temperature by alcohol catalytic chemical vapor deposition using $\mathrm{Ru}$ catalysts. MRS Advances, 1-7. doi:10.1557/adv.2018.26. 